\title{
Neurokinin-2 receptor polymorphism predicts lymph node metastasis in colorectal cancer patients
}

\author{
WEIJIA FANG $^{1 *}$, CAIYUN FU $^{2,3^{*}}$, XIAOGANG CHEN $^{1}$, XIAOZHOU MOU ${ }^{1,3}$, FANLONG LIU $^{1}$, \\ JIONG QIAN $^{1}$, PENG ZHAO ${ }^{1}$, YULONG ZHENG ${ }^{1}$, YI ZHENG ${ }^{1}$, JING DENG ${ }^{1}$, \\ PINGJIANG YE $^{4}$, YIFEI WANG ${ }^{5}$ and SHUSEN ZHENG ${ }^{1}$
}

\begin{abstract}
${ }^{1}$ Biotherapy Center, Department of Medical Oncology, The First Affiliated Hospital, School of Medicine, Zhejiang University, Hangzhou, Zhejiang 310003; ${ }^{2}$ Lab of Proteomics and Molecular Enzymology, School of Life Sciences, Zhejiang Sci-Tech University, Hangzhou, Zhejiang 310018; ${ }^{3}$ Institute for Cell-based Drug Development of Zhejiang Province, Hangzhou, Zhejiang 310058; ${ }^{4}$ Department of Anorectum, People's Hospital of Shaoxing, Shaoxing, Zhejiang 312000;

${ }^{5}$ Second People's Hospital of Jiaxing, Jiaxing, Zhejiang 314000, P.R. China
\end{abstract}

Received April 26, 2014; Accepted January 16, 2015

DOI: $10.3892 / \mathrm{ol} .2015 .3016$

\begin{abstract}
To analyze the single nucleotide polymorphisms (SNPs) of two subtypes of neurokinin (NK) receptors, $N K 1 R$ and $N K 2 R$ (also known as TAC1R and TAC2R), in colorectal cancer (CRC), peripheral blood samples were collected from 199 CRC patients. Direct-sequencing was performed to identify the NK1R rs10198644 and NK2R rs4644560 SNPs. Genotype results were correlated with clinical factors. The allele frequencies of NK1R rs10198644 GC, CC and GG were 52, 17 and 31\%, respectively, while that of $N K 2 R$ rs $4644560 \mathrm{GC}, \mathrm{CC}$, and GG were 36, 50 and $14 \%$, respectively. Patients with $N K 2 R$ rs4644560 GC exhibited more positive lymph nodes than those with $C C$ (mean, 2.2 vs. 1.3; $\mathrm{P}=0.016$ ). Further analysis highlighted that the number of positive lymph nodes was also increased in the $N K 2 R$ rs4644560 GC/NK1R rs10198644 GG group compared with the $N K 2 R$ rs $4644560 \mathrm{GG} / N K 1 R$ rs10198644 GG group (mean, 2.2 vs. $0.9 ; \mathrm{P}=0.04$ ). These data suggested that the $N K 2 R$ rs4644560 GC polymorphism alone or combination with $N K 1 R$ rs10198644 GG may be a promising prognostic marker of lymph node metastasis in CRC patients.
\end{abstract}

\section{Introduction}

Neurokinins (NKs) are small neuropeptides that are present in the tumor microenvironment and can act on different stages of

Correspondence to: Dr Shusen Zheng, Biotherapy Center, Department of Medical Oncology, The First Affiliated Hospital, School of Medicine, Zhejiang University, 79 Qinchun Road, Hangzhou, Zhejiang 310003, P.R. China

E-mail: shusenzheng@zju.edu.cn

*Contributed equally

Key words: neurokinin 2 receptor, single nucleotide polymorphisms, colorectal cancer, positive lymph node carcinogenesis (1). Substance P (SP) and NK A (NKA) and B (NKB) are classical members of the NK family, which exert their biological effects by activating NK receptors denoted as $N K 1 R, N K 2 R$ and $N K 3 R$ (also known as TAC1R, TAC2R and $T A C 3 R$ ) (2). SP, the endogenous ligand for $N K 1 R$, activates responses correlated with tumor growth in several human cell lines bearing $N K 1 R$ receptors (3). Moreover, NKA, the endogenous ligand for $N K 2 R$, is a good predictor of survival in patients with stage IV well-differentiated small bowel neuroendocrine neoplasms (4). Numerous tumors that express NKRs can misuse the NK-induced signaling of normal cells to promote the proliferation and survival of cancer cells, releasing cytokines and soluble mediators that favor tumor growth (5). However, the precise involvement and significance of NK in cancer pathologies remains to be fully defined.

NKRs belong to the family of seven transmembrane G-protein coupled receptors. $N K 1 R$ and $N K 2 R$ are dispersed throughout the central and peripheral nervous systems, while $N K 3 R$ is distributed mainly in the central nervous system (6). Human $N K 1 R$ and $N K 2 R$ are 407- and 398-amino acid proteins, respectively (7). NK2R is expressed in a variety of organs, including the gastrointestinal tract, while functional data suggest that $N K 2 R$ could be significant in mediating the NK-evoked smooth muscle contraction of organs (8). The prevalence of the $N K 2 R$ mRNA $\alpha$ isoform indicates that $N K 2 R$ is significantly involved in regulating human colonic functions (9).

In the United States, colorectal cancer (CRC) has the third highest incidence of new cases and the third highest rate of cancer-associated mortality, with 142,280 and 50,830 cases, respectively, in 2013 (10). It is also estimated that the mortality rate for CRC has been gradually increasing in China (11). The occurrence and development of this deadly disease may vary among patients; this may be attributed to variations in genotype patterns. Recently, a population-based epidemiological study in a Chinese population found that the NK2R rs4644560 genotype appeared to be associated with a decreased risk of CRC when combined with NKIR rs10198644 (12). This finding indicates that these two subtypes of $N K R$ gene may 
play an important role in CRC. The present study used blood samples collected from patients with CRC to clarify the potential association between the single nucleotide polymorphisms (SNP) of $N K 2 R$ rs4644560/NK1Rrs 10198644 and a range of clinical factors important in CRC.

\section{Materials and methods}

Participants. The present study was approved by the Ethics Committee of the First Affiliated Hospital, School of Medicine, Zhejiang University (Hangzhou, Zhejiang, China). Peripheral blood samples were collected from 199 patients with CRC following surgery in the First Affiliated Hospital, School of Medicine, Zhejiang University, between May and October 2013. All patients provided written informed consent.

Details of patient demographics and clinical information were recorded, including age, gender, alcohol consumption, tobacco exposure, primary tumor site, diagnostic stage, number of positive lymph nodes, differentiation grade and carcinoembryonic antigen (CEA) level. Lifestyle factors, including alcohol consumption and tobacco use, were defined as follows: An individual who consumed alcohol more than once per day for at least three months was considered as an alcohol drinker and an individual who had smoked more than one cigarette per day for over one year was considered as a tobacco user. Diagnostic stage was confirmed according to the National Comprehensive Cancer Network guidelines for CRC (13). Differentiation grade was defined as poor, moderate and well. An abnormal CEA level was defined as a level of $>5 \mathrm{ng} / \mathrm{ml}$.

Genotyping. Genomic DNA was extracted from whole blood samples using the QIAamp DNA Mini kit (Qiagen $\mathrm{GmbH}$, Hilden, Germany) according to the manufacturer's instructions. Genotyping was performed with polymerase chain reaction (PCR) amplification and direct-sequencing. Primers were designed with Primer Premier 5.0 software (PREMIER Biosoft, Palo Alto, CA, USA) and synthesized by Genewiz Biotech (Beijing, China). The primer sequences used for amplification were as follows: NK1R forward, 5'-AAAGTTGGGATCTGCTTACACT-3' and reverse, 5'-TTT CTTTTCTCTCACTGTCCCA-3'; and NK2R forward, 5'-ATG CTGCTGTGTCATCTGCT-3' and reverse, 5'-TATCTTGCC CAGGTTGGTCT-3'. The PCR was performed with $50 \mathrm{ng}$ of genomic DNA, $0.25 \mu \mathrm{M}$ of each primer, $200 \mu \mathrm{M}$ of each deoxyribonucleotide, $3 \mathrm{mM} \mathrm{MgCl}$ and 0.2 Units of Taq DNA polymerase (Takara, Dalian, China). The PCR amplification was performed as follows: Initial denaturation at $95^{\circ} \mathrm{C}$ for $5 \mathrm{~min}$, followed by 9 cycles of denaturation at $95^{\circ} \mathrm{C}$ for $45 \mathrm{sec}$, annealing at $58^{\circ} \mathrm{C}$ for $45 \mathrm{sec}$ (decreasing by $0.2^{\circ} \mathrm{C}$ every cycle) and elongation at $72^{\circ} \mathrm{C}$ for $30 \mathrm{sec}$; then 34 cycles of denaturation at $95^{\circ} \mathrm{C}$ for $45 \mathrm{sec}$, annealing at $55^{\circ} \mathrm{C}$ for $45 \mathrm{sec}$ (decreasing by $0.2^{\circ} \mathrm{C}$ every cycle) and elongation at $72^{\circ} \mathrm{C}$ for $30 \mathrm{sec}$; and a final elongation at $72^{\circ} \mathrm{C}$ for $5 \mathrm{~min}$.

The PCR products were purified using the SAP/EXO PCR kit (New England Biolabs, Inc., Ipswich, MA, USA). Two microliters of the purified amplicons were directly used for a sequencing reaction with the Big Dye Terminator cycle sequencing mix v3.1 (Applied Biosystems Life Technologies, Foster City, CA, USA). Dye purification was performed by alcohol/sodium acetate precipitation. Sequence analysis was
Table I. Patient characteristics $(\mathrm{n}=199)$.

Characteristic

Number $(\%)$

Gender
Male
Female

Location of primary tumor

Left colon

Right colon

Rectum

Positive lymph nodes

$0-1$

2-3

4-6

$\geq 7$

Alcohol drinker

Yes

No

Tobacco user

Yes

No

performed on an ABI 3730xl DNA analyzer (Applied Biosystems Life Technologies).

Statistical analysis. SPSS version 20.0 (SPSS, Inc., Chicago, IL, USA) was used for the analysis of the final results. $\mathrm{P}<0.05$ was considered to indicate a statistically significant difference. A two-tailed t-test (double samples heteroscedastic hypothesis) was used to clarify the association between the number of positive lymph nodes and tobacco exposure. Pearson's correlation was used to analyze the association between the number of positive lymph nodes and diagnostic stage. Bartlett's $\chi^{2}$ test was used to analyze the association between the number of positive lymph nodes and the genotypes of each NKR. The data was assessed for homogeneity of variance following logarithmic transformation.

A one-way analysis of variance (ANOVA) was used to further clarify the impact of different genotype combinations of $N K 2 R$ and $N K 1 R$ on the number of positive lymph nodes. Prior to the analysis, the genotypes of $N K 2 R$ and $N K 1 R$ were first mixed into nine groups (Fig. 1).

\section{Results}

A total of 199 patients with pathologically confirmed CRC were enrolled in the study following surgical resection. The median age of the patients was 61 years (range, 23-84 years), and the detailed patient characteristics are listed in Table I. The allele frequencies of NKIR rs10198644 GC, CC and GG were 52, 17 and 31\%, respectively, while for $N K 2 R$ rs $4644560 \mathrm{GC}, \mathrm{CC}$ and GG, the frequencies were 36,50 and $14 \%$. A comprehensive analysis of all patient factors, including demographic, lifestyle and clinical factors, was performed in order to clarify their association with the genotypes of $N K 1 R$ and $N K 2 R$, as well as the interaction between them. The positive outcomes are described below. 
Table II. Association between tobacco use and the number of positive lymph nodes.

\begin{tabular}{|c|c|c|c|c|}
\hline Parameter & Tobacco & Non-tobacco & T-value & P-value (two-tailed) \\
\hline Mean number of positive lymph nodes & 3.2 & 2.0 & 2.10 & $0.04^{\mathrm{a}}$ \\
\hline Square deviation & 16.5 & 3.1 & & \\
\hline Number of patients & 53 & 138 & & \\
\hline
\end{tabular}

${ }^{\mathrm{a}} \mathrm{P}<0.05$

Table III. Comparison of lymph nodes between the three genotypes of $N K 2 R$ rs 4644560 .

\begin{tabular}{lcc}
\hline Genotypes & Number of patients & Mean number of positive lymph nodes \\
\hline GC & 63 & $2.2^{\mathrm{a}}$ \\
CC & 79 & $1.3^{\mathrm{a}}$ \\
GG & 23 & 1.6 \\
F-value & & 4.27 \\
P-value & & $0.016^{\mathrm{a}}$ \\
\hline
\end{tabular}

${ }^{\mathrm{a}} \mathrm{P}<0.05$.

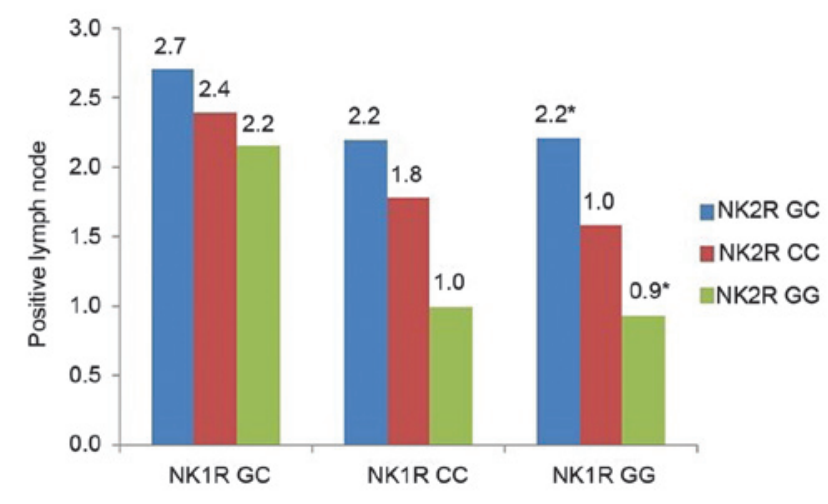

Figure 1.Comparison of median positive lymph node numbers within nine subtypes of $N K 2 R$ and NK1R combinations. "P<0.05 NK2R GC/ NK1R GG vs. NK2R GG/ NK1R GG (P=0.04).

In total, there were 191 patients for whom information regarding positive lymph node numbers and tobacco exposure was available. Among these patients, 53 were in the tobacco group and 138 were in the non-tobacco group, and the mean number of positive lymph nodes was 3.2 and 2.0, respectively. The t-test showed that the tobacco group exhibited significantly more positive lymph nodes than the non-tobacco group ( $\mathrm{P}=0.04$; Table II).

In addition, among the 196 patients for whom information on stage and number of positive lymph nodes was available, Pearson's correlation found a positive correlation between the number of positive lymph nodes and the diagnostic stage of the tumor $(\mathrm{r}=0.48 ; \mathrm{P}<0.001)$.

Finally, information pertaining to the number of positive lymph nodes and the NKR genotypes was available in 165 patients. Bartlett's $\chi^{2}$ analysis showed that
$N K 2 R$ rs4644560 GC was associated with significantly more positive lymph nodes than rs4644560 CC (mean, 2.2 vs. 1.3; $\mathrm{P}=0.016$; Table III). Furthermore, the one-way ANOVA highlighted the interaction between $N K 2 R$ rs4644560 and NK1R rs10198644, and showed that the number of positive lymph nodes was increased significantly in the rs4644560 GC/rs10198644 GG group compared with the rs4644560 GG/rs10198644 GG group (mean, 2.2 vs. 0.9; $\mathrm{P}=0.04)$, as shown in Fig. 1.

\section{Discussion}

Based on the identification of the role of NK pathway genes in the risk of CRC, the allele frequencies of SNPs in two selected subtypes of $\mathrm{NK}$ receptor, $N K 1 R$ and $N K 2 R$, were investigated and their potential association with various clinical factors was analyzed. Notably, despite the limitation of the sample size, tobacco exposure, diagnostic stage and the selected $N K R$ genotypes were all found to be significantly associated with the number of metastatic lymph nodes, which is well known to be one of the most important prognostic factors in CRC $(14,15)$.

SNPs in receptor-encoded genes can affect a number of aspects of receptor function (16), and may also result in an increased susceptibility to disease or produce variable responses to therapeutic agents (17). There are several novel gene polymorphisms, including those for interleukin-10, SDF1 $\alpha$ and embryonic ectoderm development, which have been proven to be associated with lymph node metastasis in CRC $(18,19)$.

It is well known that lifestyle factors, such as cigarette smoking and alcohol consumption, may contribute to the risk of cancer. In the present study, patients exposed to tobacco exhibited significantly more positive lymph nodes than those patients not exposed to tobacco; this result is consistent with the finding that tobacco exposure has a detrimental effect on 
CRC (20). In agreement with a previously published study (21), the present study also found that diagnostic stage was associated with the number of metastatic lymph nodes.

As aforementioned, metastatic lymph nodes may be associated with a variety of clinical factors, therefore, its potential correlation with NK receptor polymorphisms, and their mutual interactions, was worthy of investigation. A previous study has shown that the stimulation of $N K 2 R$ leads to partial blunting of the enhanced stimulatory effects mediated by $N K 1 R$. By contrast, stimulatory hematopoietic cytokines upregulate $N K 1 R$ expression and downregulate the constitutively expressed $N K 2 R$ in bone marrow stroma (22). In the present study, the role of $N K 2 R$ rs4644560 GC in predicting lymph node metastasis was clarified based on the finding that this heterozygote is associated with more positive lymph nodes than the homozygote (CC) of $N K 2 R$ rs4644560. Notably, despite the complexity of information on clinical interactions, the interactive model of $N K 2 R$ and $N K 1 R$ was also found to be useful. NK2R rs4644560 GC was associated with more positive lymph nodes than the corresponding GG genotype when combined with $N K 1 R$ rs10198644 GG. Further research should be performed to elucidate the potential mechanism underlying this interaction.

Little has been known with regard to the mechanism of $N K 2 R$ in CRC carcinogenesis. One study, however, began to shed light on this area. The study found that activation of $N K 2 R$ in afferent neurons led to the protein kinase C (PKC)-induced phosphorylation of a certain gene (23). PKC $\delta$ was known to negatively modulate the canonical Wnt pathway and cell proliferation in colon tumor cell lines (24). Moreover, the connection between $N K 2 R$ and PKC was confirmed by another study, which showed that stimulation of $N K 2 R$ was not only linked to PKC activation, but that it was also associated with the activation of a multidrug resistance protein transporter in vivo (25). Extensive reviews have also described the neuropeptide receptors as targets for the treatment and diagnosis of cancer (5). Based on the present findings, one novel therapeutic strategy for colon cancer in the future may be the inhibition of the potential signaling pathways associated with certain NK receptor genotypes.

Pending further research to clarify the potential mechanism of the malignant inclination of the $N K 2 R$ rs $4644560 \mathrm{GC}$ genotype or its combination with $N K 1 R$ rs10198644 GG, the present study indicates that these genotypes may act as a promising prognostic marker, which may predict lymph node metastasis in CRC patients.

\section{Acknowledgements}

The authors would like to thank Professor Ping Fang from Zhejiang University (Hangzhou, Zhejiang, China) for providing statistical technology support, and Aparna Rao (Peter MacCallum Cancer Centre) who provided so much assistance with perfecting the language of the manuscript and supplied useful suggestions and comments. This study was supported by the Major Scientific Project of Zhejiang Province (grant no. 2012C13014-2). The authors would also like to express their gratitude to the Consulting Project of the Chinese Academy of Engineering (grant no. 2012-XY-12-4), the National Natural Science Foundation of China (grant nos. 81201557, 81201783, 81272679, 81372463 and 31000496), the Zhejiang Natural Science Foundation (grant no. LY13H160007) and the Zhejiang Medicines and Health Science and Technology Project (grant no. 201348801) for their support.

\section{References}

1. Palma C: Tachykinins and their receptors in human malignancies. Curr Drug Targets 7: 1043-1052, 2006.

2. Severini C, Improta G, Falconieri-Erspamer G, et al: The tachykinin peptide family. Pharmacol Rev 54: 285-322, 2002.

3. Mayordomo C, García-Recio S, Ametller E, et al: Targeting of substance $\mathrm{P}$ induces cancer cell death and decreases the steady state of EGFR and Her2. J Cell Physiol 227: 1358-1366, 2012.

4. Diebold AE, Boudreaux JP, Wang YZ, et al: Neurokinin A levels predict survival in patients with stage IV well differentiated small bowel neuroendocrine neoplasms. Surgery 152: 1172-1176, 2012.

5. Reubi JC: Peptide receptors as molecular targets for cancer diagnosis and therapy. Endocr Rev 24: 389-427, 2003.

6. Quartara L and Maggi CA: The tachykinin NK1 receptor. Part II: Distribution and pathophysiological roles. Neuropeptides 32: $1-49,1998$.

7. Pennefather JN, Lecci A, Candenas ML, et al: Tachykinins and tachykinin receptors: a growing family. Life Sci 74: 1445-1463, 2004.

8. Lecci A, Santicioli P and Maggi CA: Pharmacology of transmission to gastrointestinal muscle. Curr Opin Pharmacol 2: 630-641, 2002.

9. Jaafari N, Hua G, Adelaide J, et al: Expression of the tachykinin receptor mRNAs in healthy human colon. Eur J Pharmacol 599: 121-125, 2008.

10. Siegel R, Naishadham D and Jemal A: Cancer statistics, 2013. CA Cancer J Clin 63: 11-30, 2013.

11. Wu M, Zhang SW, Han RQ, et al: Analysis on the mortality of colorectal and anal cancer in china during 2004-2005. Zhonghua Yu Fang Yi Xue Za Zhi 44: 403-407, 2010 (In Chinese).

12. Yu Y, Pan Y, Jin M, et al: Association of genetic variants in tachykinins pathway genes with colorectal cancer risk. Int J Colorectal Dis 27: 1429-1436, 2012.

13. Benson AB 3rd, Venook AP, Bekaii-Saab T, et al: Colon cancer, version 3.2014. J Natl Compr Canc Netw 12: 1028-1059, 2014.

14. Zhang B, Lv M, Chen T, et al: The association between lymph node resection and postoperative survival in patients with colorectal cancer. Hepatogastroenterology 60: 1922-1926, 2013.

15. Yuan Y, Li MD, Hu HG, et al: Prognostic and survival analysis of 837 Chinese colorectal cancer patients. World J Gastroenterol 19: 2650-2659, 2013.

16. Tang CM and Insel PA: Genetic variation in G-protein-coupled receptors - consequences for G-protein-coupled receptors as drug targets. Expert Opin Ther Targets 9: 1247-1265, 2005.

17. Castro FA, Försti A, Buch S, et al: TLR-3 polymorphism is an independent prognostic marker for stage II colorectal cancer. Eur J Cancer 47: 1203-1210, 2011.

18. Chang SC, Lin PC, Yang SH, et al: SDF-1alpha G801A polymorphism predicts lymph node metastasis in stage T3 colorectal cancer. Ann Surg Oncol 16: 2323-2330, 2009.

19. Seo GS, Yu JI, Chae SC, et al: EED gene polymorphism in patients with colorectal cancer. Int J Biol Markers 28: 274-279, 2013.

20. Araujo RF Jr, Lira GA, Guedes HG, et al: Lifestyle and family history influence cancer prognosis in Brazilian individuals. Pathol Res Pract 209: 753-757, 2013.

21. Noura S, Ohue M, Kano S, et al: Impact of metastatic lymph node ratio in node-positive colorectal cancer. World J Gastrointest Surg 2: 70-77, 2010.

22. Rameshwar P, Poddar A and Gascón P: Hematopoietic regulation mediated by interactions among the neurokinins and cytokines. Leuk Lymphoma 28: 1-10, 1997.

23. Sculptoreanu A, Aura Kullmann F and de Groat WC: Neurokinin 2 receptor-mediated activation of protein kinase C modulates capsaicin responses in DRG neurons from adult rats. Eur J Neurosci 27: 3171-3181, 2008.

24. Hernández-Maqueda JG, Luna-Ulloa LB, Santoyo-Ramos P, et al: Protein kinase $\mathrm{C}$ delta negatively modulates canonical Wnt pathway and cell proliferation in colon tumor cell lines. PLoS One 8: e58540, 2013.

25. Sun J, Usune S, Zhao Y, et al: Multidrug resistance protein transporter and Ins $(1,4,5) \mathrm{P}_{3}$-sensitive $\mathrm{Ca}^{2+}$-signaling involved in adenosine triphosphate export via Gq protein-coupled $\mathrm{NK}_{2}$-receptor stimulation with neurokinin A. J Pharmacol Sci 114: 92-98, 2010. 